\title{
On the scaling relations of disk galaxies
}

\author{
Riccardo Giovanelli \\ Department of Astronomy, Cornell University, Space Sciences Building, Ithaca, NY 14853, \\ USA \\ email: rg39@cornell.edu
}

\begin{abstract}
The physical background of scaling laws of disk galaxies is reviewed. The match between analytically derived and observed scaling laws is briefly discussed. Accurate modeling of the fraction of baryons that end up populating a disk, and the conversion efficiency of those into stars, remains a challenging task for numerical simulations. The measurement of rotational velocity tends to be made with criteria of convenience rather than through rigorous definition. And yet, the Tully-Fisher and the disk size versus rotational velocity relations exhibit surprisingly small scatter. Practical recipes (and costs) to optimize the quality of template relations are considered.
\end{abstract}

Keywords. galaxies: distances and redshifts, galaxies: halos, galaxies: spiral, distance scale

\section{Introduction}

Thirty-five years have passed since Brent Tully and Rick Fisher advocated the use of the relation between optical luminosity, $L$, and $21 \mathrm{~cm}$ Hi linewidth, $W$, previously discovered by Mort Roberts, as a tool to obtain redshift-independent distances to spiral galaxies (Roberts 1969; Tully \& Fisher 1977). Since then, the Tully-Fisher relation (hereafter TFR) has been used extensively in measurements of $\mathrm{H}_{0}$ as well as of deviations from a smooth Hubble flow, to distances approaching $z \simeq 0.1$. Over the range of linewidths $W>100 \mathrm{~km} \mathrm{~s}^{-1}$, typically used in those applications, the TFR exhibits a tight power-law behavior, where the linewidth is (to first order) equal to twice the maximum rotational velocity of the disk within galactocentric radii populated with detectable $\mathrm{HI}$, and $L$ is a proxy for the total mass within that radius. A TFR template will have a slope of between 3 and 4 in $\log L$ versus $\log W$ and a scatter between 0.14 and $0.18 \operatorname{dex}$ in $\log L$ (0.35 to 0.45 mag), which translates to a distance uncertainty for a single galaxy between 15 and $20 \%$. These values vary somewhat depending mostly on the adopted optical band. The quality of a TFR template, i.e. the accuracy with which it can accurately provide a distance prediction, depends on a variety of parameters, including primarily those pertaining to the selection criteria for membership in the sample used to define that template and the reliability of the corrections applied to the observed quantities. Improvements to that quality should result from a clear understanding of the sources of scatter and of the physical basis of the TFR.

\section{Towards a Physical Understanding of the TFR}

Take 1. Assume that disk mass and light are exponentials with scale length $r_{\mathrm{d}}$. The luminosity is then $L_{\mathrm{d}} \propto r_{\mathrm{d}}^{2} I(0)$, where $I(0)$ is the disk's central surface brightness. The mass within radius $r$ is $M(<r) \propto r v_{\text {rot }}^{2}$, so if the rotation curve becomes flat due to a dark matter (DM) halo truncated at $n$ scale lengths, then $M_{\text {tot }} \propto n r_{\mathrm{d}} v_{\max }^{2}$ and

$$
L_{\mathrm{d}} \propto a v_{\max }^{4}, \text { with } a=\left(M_{\mathrm{d}} / L_{\mathrm{d}}\right)^{-2} m_{\mathrm{d}}^{2} n^{-2} I(0)^{-2},
$$


where $M_{\mathrm{d}}\left(m_{\mathrm{d}}\right)$ is the mass (fraction) of the disk. A rough match for the slope of the TFR is recovered, but much is contained in $a$.

Take 2. Mo et al. (1998) model the DM halo, within which the disk galaxy resides, as a singular isothermal sphere. Then the rotational velocity, $v_{\mathrm{c}}$, is the same at all radii, and the mass density at, and the mean density within, radius $r$ are

$$
\rho(r)=\frac{v_{\mathrm{c}}^{2}}{4 \pi G r^{2}} \quad \text { and } \quad \bar{\rho}=\frac{3 v_{\mathrm{c}}^{2}}{4 \pi G r^{2}} .
$$

The critical density of the Universe at redshift $z$ is

$$
\rho_{\text {crit }}=\frac{3 H^{2}(z)}{8 \pi G},
$$

so the radius within which the mean density is $200 \rho_{\text {crit }}$ is $r_{200}=v_{\mathrm{c}} / 10 H(z)$ and the mass within $r_{200}$ is

$$
M_{\text {halo }}=v_{\mathrm{c}}^{2} r_{200} / G=v_{200}^{3} / 10 G H(z) .
$$

Introducing the disk mass, $M_{\mathrm{d}}$, and $m_{\mathrm{d}}=M_{\mathrm{d}} / M_{\text {halo }}$, we get

$$
M_{\mathrm{d}}=\frac{m_{\mathrm{d}} v_{200}^{3}}{10 G H(z)} \simeq 1.7 \times 10^{11} h^{-1} \mathrm{M}_{\odot}\left(\frac{m_{\mathrm{d}}}{0.05}\right)\left(\frac{v_{200}}{250 \mathrm{~km} \mathrm{~s}^{-1}}\right)^{3}\left[\frac{H(z)}{H_{0}}\right]^{-1} .
$$

This is starting to look familiar, but:

- halos are not singular isothermal spheres;

- disk masses are not negligible and they can alter the total density profile;

- we measure disk luminosity, not disk mass;

- we do not measure $v_{200}$ but rather a circular (?) velocity at whatever radius Nature kindly leaves a tracer for us to measure.

Take 3. We thus abandon the singular isothermal sphere model and, still following Mo et al. (1998), model the mass density more realistically with the Navarro, Frenk, \& White (NFW) profile:

$$
\rho(r)=\frac{\rho_{\text {crit }} \delta_{0}}{\left(r / r_{\mathrm{s}}\right)\left(1+/ r_{\mathrm{s}}\right)^{2}},
$$

where $\delta_{0}=4 \rho\left(r_{\mathrm{s}}\right) / \rho_{\text {crit }}$ and $c=r_{200} / r_{\mathrm{s}}$ is the concentration index. The mass within radius $r$ is then

$$
M(r)=4 \pi \rho_{\text {crit }} \delta_{0} r_{\mathrm{s}}^{3}\left[\frac{1}{1+c\left(r / r_{200}\right)}-1+\ln \left(1+r / r_{200}\right)\right],
$$

the total halo mass is

$$
M_{\text {halo }}=4 \pi \rho_{\text {crit }} \delta_{0} r_{\mathrm{s}}^{3}[\ln (1+c)-c /(1+c)],
$$

and the rotation curve is given by

$$
\frac{v(r)}{v_{200}}=\left[\frac{1}{x} \frac{\ln (1+c x)-c x /(1+c x)}{\ln (1+c)-c /(1+c)}\right]^{1 / 2},
$$

where $x=r / r_{200}$ and $v_{200}=\left(G M_{\text {halo }} / r_{200}\right)^{1 / 2}$.

Fig. 1 (after Mo et al. 1998) shows rotation curves of galaxies characterized by the same disk mass but different combinations of concentration indices, disk mass fractions, and spin parameters. The shape of the rotation curve, its maximum value, and its value at the virial radius (indicated by the short, thick line at the left-hand side of each box) vary widely, illustrating the uncertainty that can be associated with the determination of 


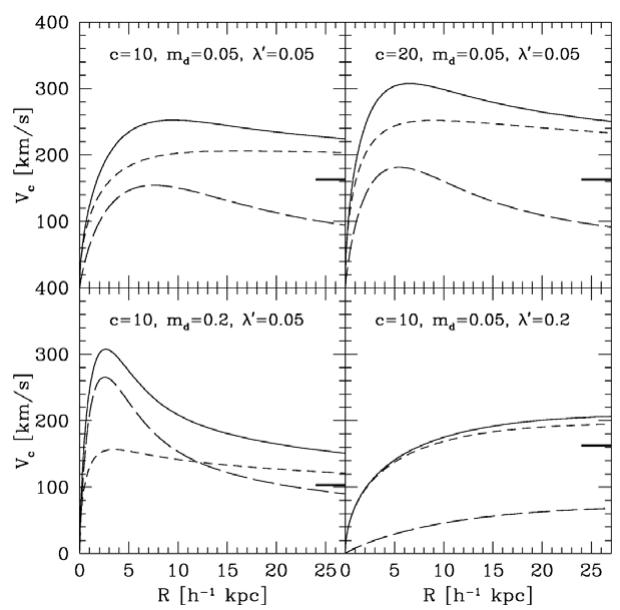

Figure 1. NFW rotation curves of galaxies having the same disk mass $\left(5 \times 10^{10} h^{-1} \mathrm{M}_{\odot}\right)$ and different combinations of concentration indices, disk mass fractions, and spin parameters, as indicated in the legends within each box. Disk and halo contributions to the rotation curve (solid line) are shown as, respectively, long- and short-dashed lines. The thick line on the right of each panel is the circular velocity, $v_{200}$, at the virial radius. Note the variance in the shapes of the rotation curves and in their amplitudes at any radius. (Credit: Mo et al. 1999)

the TFR linewidth parameter. Because now $v_{\text {rot }}(r)$ is not constant, it matters at which radius $r_{\text {meas }}$ it is measured. It is sensible to measure it at a radius near which the gradient of $v_{\text {rot }}$ is small, e.g., the radius within which $80 \%$ of the light is produced, or a few disk scale lengths out, $n r_{\mathrm{d}}$ (a value of $n \simeq 3$ is often used, and $r_{80}$ is near $3 r_{\mathrm{d}}$ ). The equation for $M_{\mathrm{d}}$ is then modified via a fudge factor which accounts for the shape of $v_{\text {rot }}$ and the radius at which it is measured. For instance, if $r_{\text {meas }}=3 r_{\mathrm{d}}$, then that fudge factor is $f_{v}=\left[v_{\text {rot }}\left(3 r_{\mathrm{d}}\right) / v_{200}\right]^{-3}$. Finally, to convert the disk mass relation into a TFR look-alike, $M_{\mathrm{d}}$ is divided by a stellar mass-to-luminosity ratio; a star-formation efficiency factor, $\epsilon_{\mathrm{sf}}$, is also introduced, which accounts for the fraction of the disk's baryon mass which has been converted to stars. Thus,

$$
L_{\mathrm{d}}=1.7 \times 10^{11} h^{-1} \mathrm{M}_{\odot} \epsilon_{\mathrm{sf}}\left(\frac{m_{\mathrm{d}}}{0.05}\right)\left(\frac{M}{L}\right)_{*}^{-1}\left[\frac{v_{\mathrm{obs}}\left(3 r_{\mathrm{d}}\right)}{250 \mathrm{~km} \mathrm{~s}^{-1}}\right]^{3}\left[\frac{H(z)}{H_{0}}\right]^{-1} f_{v} .
$$

Accurately modeling $m_{\mathrm{d}} \epsilon_{\mathrm{sf}}(M / L)_{*}^{-1}$ is difficult, and so is measuring $f_{v}$. Moreover, the value of the rotation curve at $3 r_{\mathrm{d}}$ may not be known from the kinematic data. Much progress has been made since Steinmetz \& Navarro (1999) in modeling the gastronomy of disks, thanks to increased resolution of numerical simulations and a higher grade of sophistication in treating feedback mechanisms, including adiabatic contraction of the halo (e.g., Governato et al. 2007; Piontek \& Steinmetz 2011). Generally, simulations recover the slope of the TFR, but have problems in reproducing the photometric zero point. In particular, the stellar mass fraction in hydrodynamical simulations tends to be too high and bulges too large, while galaxies at low $z$ are too gas-poor (Scannapieco et al. 2012). Improvements are obtained by forcing a slow-down in the growth of bulges. However, processes of feedback, those affecting interstellar medium (ISM) structure and metallicity, and their effect on star formation take place on scales which are still much smaller than those currently achievable by cosmological simulations. As for $f_{v}$, an interesting observational result has recently come to the fore, throwing additional light on Eq. (2.10), as we will see next. 

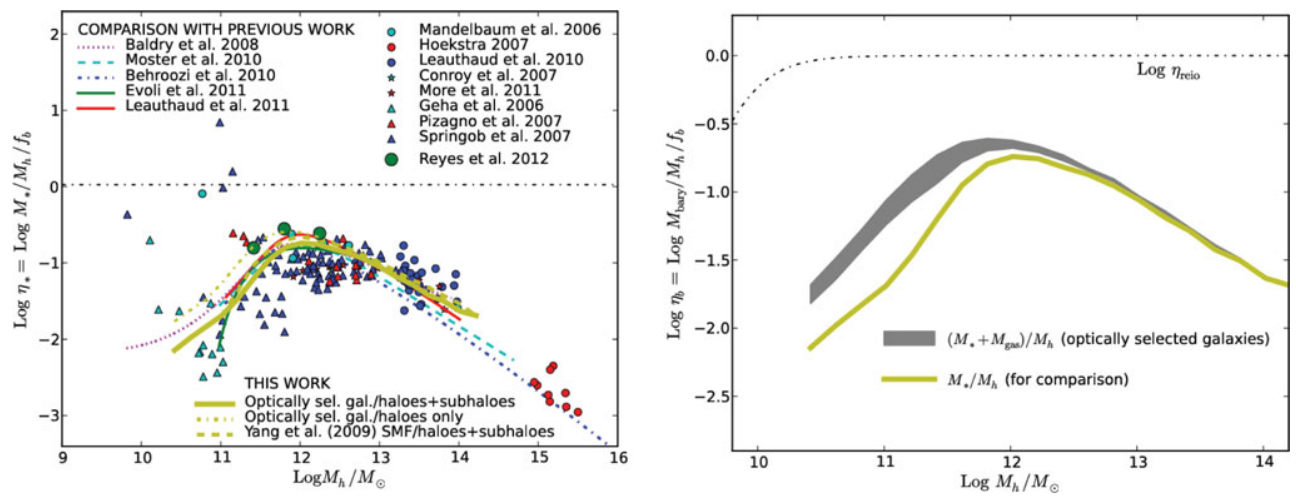

Figure 2. (left) Stellar mass as a fraction of the baryon mass within the halo, plotted versus the halo mass; the baryon mass within the halo is $f_{\mathrm{b}} M_{\mathrm{halo}}$, where $f_{\mathrm{b}}$ is the cosmic baryon fraction. (Credit: Papastergis et al. 2012) (right) Observable baryon mass (stars plus disk ISM) as a fraction of the total baryon mass within the halo, plotted versus the total halo mass (shaded curve). The thin line is the stellar mass fraction, as in the left-hand panel. (Credit: Papastergis et al. 2012. Reproduced with permission from the American Astronomical Society)

Reyes et al. (2012) directly measured the average $f_{v}$ value, implementing a technique earlier adopted by Seljak (2002). They used the sDss database, stacking images of 133,598 disk-galaxy images at a mean $z=0.07$, in three bins of stellar mass. The signature of weak lensing was identified in the stacked images, thus yielding average halo masses out to $r_{200}$. The stellar mass bins were centered respectively at $M_{*}=0.6,2.7$, and $6.5 \times 10^{10}$ $\mathrm{M}_{\odot}$. They could then measure $M_{200}$ and $r_{200}$, and hence $v_{200}$. From a child catalog of galaxies with resolved rotation curves, they obtained $v_{\mathrm{obs}}\left(r_{80}\right)$; then the averages of the ratios $v_{\text {obs }}\left(r_{80}\right) / v_{200}$, for the three stellar mass bins, were found to be respectively $1.27,1.39$, and 1.27. Interestingly, the corresponding stellar masses, as fractions of the total baryon mass $f_{\mathrm{bar}} M_{200}$, computed assuming baryons are present with the cosmic abundance within the halo, are $0.15,0.26$, and 0.23 , respectively, for the three stellar mass bins, where $f_{\mathrm{bar}}=0.169$ is the cosmic baryon mass fraction. Disk galaxies in the three stellar mass bins fall short of converting their baryons into stars by factors of between 4 and 5. This is illustrated graphically in Fig. 2 (after Papastergis et al. 2012). In each panel, the abscissa is the halo mass; in the left-hand panel, the ordinate is the ratio of the stellar mass to $f_{\mathrm{bar}} M_{200}$; in the right-hand panel, the ordinate is the ratio of the combined baryon mass of stars and cold disk gas to $f_{\mathrm{bar}} M_{200}$. The mismatch between the observed baryon mass in disks and that which would be expected if the baryons were present within the halo in the fraction $f_{\text {bar }}$ is thought to be mainly due: (i) for low halo masses, to the inability of the halos to hold on to their baryons, which increases with decreasing halo mass; and (ii) for galaxies near the peak of the curve, likely to the fact that in those systems most baryons are in hot galactic coronae, rather than in disks. The Reyes et al. (2012) data are identified by the three circles near the top of the curve in the left-hand panel.

Eq. (2.10) and Fig. 2 summarize our understanding of the physical basis of the TFR. It remains surprising that the variance in the combination of parameters $m_{\mathrm{d}} \epsilon_{\mathrm{sf}}(M / L)_{*}^{-1} f_{v}$ is as small as indicated by the observed scatter in the TFR, and that the baryonic version of the TFR remains a pure power law over five orders of magnitude in mass (see Fig. 4 and Section 4). 


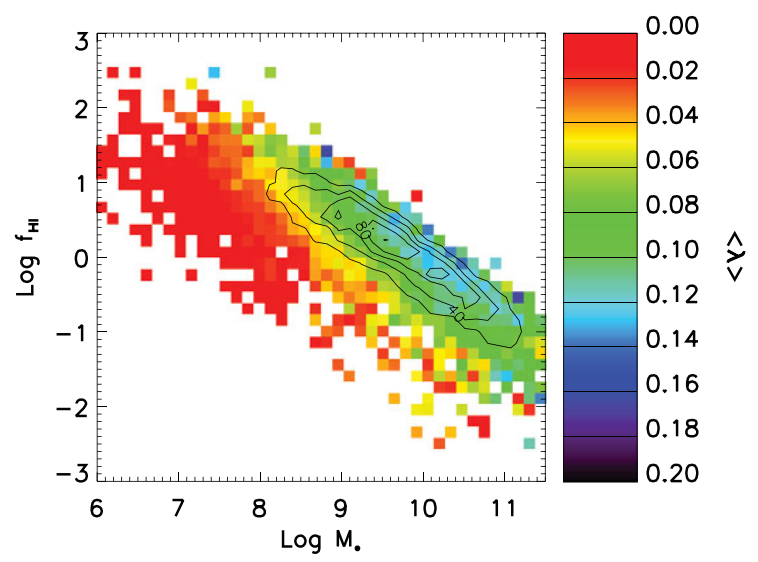

Figure 3. HI gas fraction $f_{\mathrm{HI}}=M_{\mathrm{HI}} / M_{*}$ of 7459 ALFALFA survey galaxies versus stellar mass, $M_{*}$; shading (color) represents the mean value of the spin parameter $\lambda$ within each $\left(\log f_{\mathrm{HI}}, \log M_{*}\right)$ cell. Cells in the diagram containing more than 20 galaxies fall within the region bounded by contour lines. The typical galaxy used for the TFR in cosmic distance studies falls within a few highly populated cells (those within the contour lines) in the diagram with $\log M_{*}>10$ and $\log f_{\mathrm{HI}}>0.1$. As Huang et al. (2012) point out, the mean value of $\lambda$ remains nearly constant along lines of constant $\log M_{\mathrm{HI}}$; see section 3.1 for discussion. (Credit: Huang et al. 2012. Reproduced with permission from the American Astronomical Society)

\section{Understanding Sources of Scatter: the $R V$ Scaling Law}

An interesting case study on the sources of scatter is that of the disk size versus $v_{\text {obs }}$ (for short, $R V$ ) scaling law. Assuming that the baryons collapse within the potential well of the halo without altering its density profile, radiating but conserving angular momentum, and settling into a thin, exponential disk of scale length $r_{\mathrm{d}}$, a reasoning similar to that described in the previous section yields

$$
r_{\mathrm{d}} \simeq 8.8 h^{-1}\left(\frac{\lambda}{0.05}\right)\left(\frac{j_{\mathrm{d}}}{m_{\mathrm{d}}}\right)\left(\frac{v_{\mathrm{c}}}{250 \mathrm{~km} \mathrm{~s}^{-1}}\right)\left[\frac{H(z)}{H_{0}}\right]^{-1} f_{\mathrm{c}} f_{\mathrm{R}} \mathrm{kpc},
$$

where $\lambda$ is the spin parameter of the halo, $j_{\mathrm{d}}$ the fraction of the angular momentum carried by the disk, $f_{\mathrm{c}}$ a fudge factor which depends solely on the concentration index of the NFW halo profile, and $f_{\mathrm{R}}$ a factor which depends mainly on the shape of the rotation curve.

An important source of scatter in this relation would appear to be that driven by the variance in $\lambda . N$-body simulations show that, over all halos, $\lambda$ has a lognormal distribution centered near 0.045 with a scatter of $0.22 \operatorname{dex}$ in $\log \lambda$. Thus, it would be expected that the observed scatter of the $R V$ law is greater than 0.22 dex in $\log r_{\mathrm{d}}$. Yet, the observed scatter is no greater than 0.16 dex (Courteau et al. 2007; Saintonge \& Spekkens 2011). The possible explanation of the mismatch is found in fig. 14 of Huang et al. (2012), reproduced here as Fig. 3. Using the $\alpha .40$ release of the ALFALFA survey (Haynes et al. 2011), Huang et al. derive spin parameters of 7459 galaxies using the estimator

$$
\lambda=21.8 \frac{r_{\mathrm{d}}[\mathrm{kpc}]}{v_{\text {rot }}^{3 / 2}\left[\mathrm{~km} \mathrm{~s}^{-1}\right]} .
$$

The inferred values of $\lambda$ are very coarse, and their dispersion is, as a result, much broader than the intrinsic spread. In a plane of HI gas fraction, $f_{\mathrm{HI}}=M_{\mathrm{HI}} / M_{*}$, versus stellar mass, $M_{*}$, the average value of $\lambda$ within each cell of that plane is represented by a different degree of shading (color). Cells containing more than 20 galaxies fall within 
the region bounded by contour lines. The typical galaxy used by the TFR in cosmic distance studies falls within a few highly populated cells (those within the contour lines) of the diagram, stretching along lines of roughly constant HI mass in the region with $\log M_{*}>9.5\left[\mathrm{M}_{\odot}\right]$ and higher-than-average $\log f_{\mathrm{HI}}$ for any given $M_{*}$. Because the $\lambda$ estimator used is a ragged one, an estimate of the intrinsic $\log \lambda$ scatter is difficult to derive. However, as Huang et al. point out, it is apparent in Fig. 3 that the mean value of $\lambda$ remains nearly constant along lines of constant $M_{\mathrm{HI}}$. Thus, the typical TFR target galaxies are extracted from a sample with tighter variance than that in any sample representative of the global halo-mass function resulting from numerical simulations. The less-than-initially-feared impact of $\lambda$ scatter on the $R V$ law makes the latter a desirable complement to the TFR. Saintonge \& Spekkens (2011) find that the scatter in the $I$-band $R V$ relation can be reduced to $\sim 0.11$ dex in the linear size parameter if $r_{\mathrm{d}}$ is replaced with an isophotal radius measured at a sufficiently large galactocentric distance so that the disk is transparent and the isophotal radius requires no correction for opacity. Such an $R V$ relation is comparable in predictive quality with the TFR. Saintonge \& Spekkens use a template $R V$ relation from the Cornell SCI data set to derive an estimate of the Hubble parameter: $\mathrm{H}_{0}=72 \pm 7 \mathrm{~km} \mathrm{~s}^{-1} \mathrm{Mpc}^{-1}$.

\section{Ways to Improve Your Scaling Law Template}

Significant benefits to a cosmic distances program based on disk galaxy scaling laws can result from wise choices in terms of template sample selection and optimization of photometric and kinematic parameters.

- Sample Selection: All Sky. The cosmic microwave background (CMB) dipole is due to the peculiar velocity of the Milky Way. It produces an apparent reflex motion, with respect to the observer, of galaxies in shells of progressively increasing radius. Asymptotically, that motion converges both in amplitude and apex direction toward the
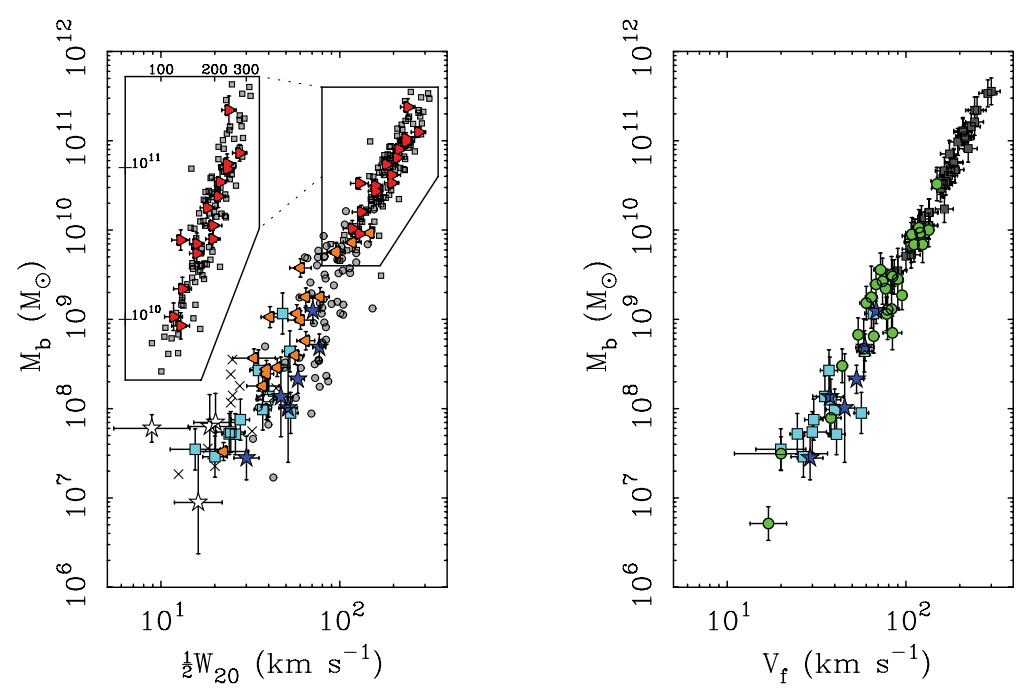

Figure 4. Baryonic mass as a function of half linewidth (left) and circular velocity, as obtained from resolved rotation curves (right). The section of the plot for the high-mass end of the relation is overplotted with higher resolution in the left-hand panel. Note (i) the power-law extension of the relation all the way to the low-mass end, and (ii) the significant reduction of the scatter when the rotational velocity is extracted from a resolved rotation curve. (Credit: McGaugh 2012. Reproduced with permission from the American Astronomical Society) 
CMB dipole vector. Convergence is reached at shell radii of $\sim 100 \mathrm{Mpc}$ (Giovanelli et al. 1998; Dale \& Giovanelli 2000). Combined with their clustering properties, an uneven distribution of galaxies in the template sample can produce TFR template relations with an incorrect zero point, simulating inflating or deflating, Local Group-centered Hubble bubbles.

- Sample Selection: Basket of Clusters. Photometric parameters of galaxies, such as inclinations to the line of sight and disk scale lengths, are best measured for relatively nearby objects. Peculiar velocities of galaxies can contribute a measurable fraction of TFR scatter at distances up to $\simeq 100 \mathrm{Mpc}$. In the case of a cluster with $N$ measured members, the offset from a template due to the cluster motion can be gauged with an accuracy that is approximately $\sqrt{N}$ times better than for a single galaxy. Thus, if the TFR template sample consists of galaxies in clusters, the component of the scatter introduced by each cluster's peculiar velocity is removable. This can be achieved by adding cluster galaxies to the template after their offset from the mean TFR, due to the peculiar velocity of their parent cluster, has been removed. A template relation built from galaxies in a basket of clusters will thus have smaller scatter than one obtained for a field-galaxy sample.

- Photometric Band. Because a good estimate of the kinematic parameter in the TFR requires that galaxies be sufficiently inclined to the line of sight, corrections to the optical flux for internal extinction are an important source of TFR scatter. This advises for selecting a photometric band within which internal extinction corrections are small, a concern that needs to be weighed against the requirement that the photometry be as representative as possible of the stellar population. Major TFR surveys of the last two decades chose either the $R$ or $I$ optical bands as a compromise between the two requirements. More recently, the wide field coverage of the Spitzer Space Telescope's archival data in the $3.8 \mu \mathrm{m}$ band has offered the opportunity to build substantial TFR samples for which extinction corrections - very small - and homogeneity of the photometric source offer the possibility of reduced scatter. The recent work of Sorce et al. (2012) indicates that, while the reliance on a consistent photometric scale over the entire sky is a definite plus, improvements in TFR scatter by going to the mid-IR regime are not yet substantial: the scatter of a preliminary $3.8 \mu \mathrm{m}$ template is $0.49 \mathrm{mag}$, which can be reduced to 0.42 mag after introducing a color correction. Contamination by emission from hot dust and PAHs may play a role in the relatively large scatter in this relation.

As just indicated, a color correction can have an important impact in reducing the TFR scatter. Until recently, large TFR samples were monochromatic. A dependence of the TFR template attributed to morphological type has often been found in monochromatic TFR templates (see, e.g., figs 3 and 4 of Giovanelli et al. 1997): that is in fact a color dependence. Availability of multicolor photometry can thus be a good asset towards building a good scaling-relation template.

- Stellar versus Baryonic. In the TFR, disk luminosity is considered a proxy for stellar mass, and the relation becomes significantly noisier for disks with rotational velocities lower than $50 \mathrm{~km} \mathrm{~s}^{-1}$. However, the power-law character of the TFR is preserved through slower rotators if disk luminosity is replaced with the baryon mass, i.e. the sum of the ISM mass, as obtained through the $21 \mathrm{~cm} \mathrm{HI}$ line, and the stellar mass as inferred from the disk luminosity and color. Fig. 4 (after McGaugh 2012) shows the baryonic TFR behavior as a single power law to rotational velocities as low as $20 \mathrm{~km} \mathrm{~s}^{-1}$. The usefulness of this relation can apply to the determination of distances to nearby dwarf systems, as an economical substitute for techniques such as measuring the tip of the red giant branch.

- Linewidths versus Resolved Rotation Curves. The two panels of Fig. 4 differ in the adoption of the kinematic parameter: the scatter in the baryonic TFR is 
significantly reduced if the rotational velocity is inferred from a resolved rotation curve (the label $v_{\mathrm{f}}$ indicates that the velocity was measured at a radius where the rotation curve becomes 'flat'). A template that uses single-dish Hi linewidths is economical, but it can be improved if replaced with $\mathrm{H} \alpha$ rotation curves and, even more so, if HI synthesis maps are available, since the Hi can typically be detected to larger galactocentric radii than $\mathrm{H} \alpha$ and therefore provide better sampling of the large-scale dynamics of the galaxy.

- The Cost of Reduced Scatter. Several of the recommendations in this section require more data than does the 'basic' TFR. For the added value, there is a cost penalty. Since we live in times of fiscal austerity, it can be useful to quantify the cost of reduced scatter. The more 'expensive' observational parameter is the kinematic one. Consider three versions of the latter, most often used, and the operating costs of telescopes needed to obtain them., and see how they would impact a TFR project budget:

○ Single-dish Hi linewidths; cost: $\sim \$ 200$ galaxy $^{-1}$; feasible sample size: $\sim 10^{4}$;

- $\mathrm{H} \alpha$ rotation curve; cost: $\sim \$ 2 \mathrm{~K}_{\text {galaxy }^{-1}}$; feasible sample size: $\sim 10^{3}$;

$\circ$ Hi synthesis map; cost: $\sim \$ 15 \mathrm{~K}_{\text {galaxy }^{-1}}$; feasible sample size: $\sim 10^{2}$.

These are very rough numbers. Perhaps better deals can be had after careful shopping.

- Invitation to Harvest. The ALFALFA Hi survey is now complete and nearly half of the extragalactic Hi sources are in the public domain. The final catalog will contain more than 30,000 sources, including optical identifications, Hi masses, and linewidths (see Giovanelli et al. 2005; Haynes et al. 2011).

\section{Acknowledgements}

This work has been supported by a grant from the U.S. National Science Foundation.

\section{References}

Courteau, S., Dutton, A. A., van den Bosch, F. C., et al. 2007, ApJ, 671, 203

Dale, D. A. \& Giovaneli, R. 2000, in: Cosmic Flows Workshop (Courteau, S., \& Willick, J., eds.), Astron. Soc. Pac. Conf. Ser., 201, p. 25

Giovanelli, R., Haynes, M. P., Freudling, W., et al. 1998, ApJ, 505, L91

Giovanelli, R., Haynes, M. P., Kent, B. R., et al. 2005, AJ, 130, 2598

Governato, F., Willman, B., Mayer, L., et al. 2007, MNRAS, 374, 1479

Haynes, M. P., Giovanelli, R., Martin, A. M., et al. 2011, AJ, 142, 170

Huang, S., Haynes, M. P., Giovanelli, R., et al. 2012, ApJ, 756, 113

McGaugh, S. S. 2012, AJ, 143, 40

Mo, H. J., Mao, S., \& White, S. D. M.. 1998, MNRAS, 319, 336

Papastergis, E., Cattaneo, A., Huang, S., Giovanelli, R., \& Haynes, M. P. 2012, ApJ, 759, 138

Piontek, F. \& Steinmetz, M. 2011, MNRAS, 410, 2625

Reyes, R., Mandelbaum, R., Gunn, J. E., Nakajima, R., Seljak, U., \& Hirata, C. M. 2012, MNRAS, 425, 2610

Roberts, M. S. 1969, AJ, 74, 859

Saintonge, A., \& Spekkens, K. 2011 ApJ, 726, 77

Scannapieco, C., Wadepuhl, M., Parry, O.H., et al. 2012 MNRAS, 423, 1726

Seljak, U. 2002, MNRAS, 334, 797

Sorce, J. G., Tully, R. B., \& Courtois, H. M. 2012, AJ, 144, 133

Steinmetz, M. \& Navarro, J. F. 1999, ApJ, 513, 555

Tully, R. B. \& Fisher, J. R. 1977, A\& A, 54, 661 